\title{
CORRIGENDUM
}

\section{Molecular genetics of Rett syndrome: when DNA methylation goes}

unrecognized

\section{Thierry Bienvenu and Jamel Chelly}

Nature Reviews Genetics 7, 415-426 (2006), doi:10.1038/nrg1878

On page 422 of this Review it is incorrectly stated that: "Further evidence for MeCP2 function as a regulator of BDNF expression was provided by postmortem studies of brains from patients with Rett syndrome." In fact, no evidence has yet been provided that MeCP2 functions as a regulator of BDNF expression in the brains of patients with Rett syndrome. This error could also lead to misinterpretation of the following sentence: "BDNF levels were found to be higher in the 'Rett-syndrome-affected' prefrontal cortex and lower in the 'Rett-syndrome-spared' occipital cortex, particularly after childhood." The study that is cited analysed only tissue from healthy brains; the sentence refers to BDNF levels in the areas that would be expected to be affected in individuals with Rett syndrome. The authors apologize for the error. 
PERSPECTIVES 
PERSPECTIVES 
PERSPECTIVES 
